\title{
A RESIDUE FORMULA FOR HOLOMORPHIC VECTOR-FIELDS
}

\author{
RAOUL BOTT
}

\section{Introduction}

Let $X$ be a holomorphic vector-field on the compact complex analytic manifold $M$. In an earlier note [3], the behavior of $X$ near its zeros was related to the characteristic numbers of the tangent bundle to $M$, and the explicit form of this relation was computed in the most nondegenerate situation, that is, in the case of $X$ vanishing at isolated points to the first order. Our aim here is to extend this result in two directions. On the one hand we consider the characteristic numbers of more general bundles $E$ over $M$ on which $X$ "acts", and on the other hand we allow $X$ to vanish along submanifolds of higher dimension but still only to the first order.

Both extensions are therefore essentially technical in nature. The first extension, to more general bundles, is especially direct and is worthwhile only in so far as it helps to clarify the arguments of [3]. The extension to higher dimensional zero-sets is less immediate and also seems to me of some interest for the following reason:

When $X$ has isolated singularities the formulas in question may be derived from the generalized Lefschetz formula for transversal maps (see [2]). In the present more general case this is not so; one would first of all need a suitable generalization of the Lefschetz formula, and such an extension is available now only if $X$ satisfies some additional restrictions, such as leaving a Riemannian structure invariant. ${ }^{1}$ A Lefschetz formula for non-transversal maps is of course closely related to the Riemannian-Roch question, so that our ultimate motivation for this note is the hope that our results might be useful in an eventual purely geometric understanding of Riemann-Roch and its generalizations.

To describe our results we need to define two notions. First of all, by an

Communicated September 30, 1967. This research was partially supported by the National Science Foundation under grant GP-6585.

${ }^{1}$ In recently completed forthcoming papers by M. Atiyah \& I. Singer [1], on the one hand and Illusie [4] on the other, our formula is derived by quite different methods for vector fields of this type. Illusie also derives a mod $p$ version of our theorems for transformations of order $p$. 
"action" of the vector-field $X$ on the vector bundle $E$, we will mean a differential operator

$$
\Lambda: \Gamma(E) \rightarrow \Gamma(E)
$$

on the $C^{\infty}$ sections of $E$ which relative to $C^{\infty}$-functions $f$ satisfies the derivation identity :

$$
\Lambda(f s)=(X f) \cdot s+f \cdot \Lambda(s) .
$$

When $M, X$ and $E$ are holomorphic such an action will be called holomorphic, if in addition $\Lambda$ commutes with $d^{\prime \prime}$ - the $\partial / \partial \bar{z}$ part of the exterior derivative $d$ :

$$
d^{\prime \prime} \cdot \Lambda=\Lambda \cdot d^{\prime \prime}
$$

Thus the prototype for an action of this sort is the action of $X$ on vector fields given by the bracket operation

$$
\theta(X): Y \rightarrow[X, Y]
$$

and it was only this action which was considered in [1].

Secondly we need a convenient formalism for describing characteristic numbers etc. For this purpose we make the following conventions.

Let $\varphi\left(x_{1}, \cdots, x_{q}\right)$ be a symmetric homogeneous polynomial in $q$ variables with complex coefficients. We then define the value of $\varphi$ on an endomorphism $A: V \rightarrow V$, of a complex $q$-dimensional vector space by the formula:

$$
\varphi(A)=\varphi\left(\lambda_{1}, \lambda_{2}, \cdots, \lambda_{q}\right)
$$

where $\lambda_{i}$ are the eigenvalues of $A$.

On the other hand we may define the value $\varphi(E)$ of $\varphi$ on a complex vector bundle $E$ over $M$ to be the cohomology class of $M$ which is representative by $\varphi(x)$ when the $x_{i}$ are interpreted as the formal roots of the Chern class of $E$, that is, when the identity

$$
\prod_{i=1}^{q}\left(1+t x_{i}\right)=1+t c_{1}(E)+\cdots+t^{m} c_{q}(E)
$$

is assumed valid and is used to express the $x_{i}$ 's in terms of the Chern classes $c_{i}(E)$ of $E$.

One may finally extend and unify these two definitions in the following manner. Suppose that $E$ is a $q$-dimensional $C$-bundle over the space $M$, and that $\Lambda: E \rightarrow E$ is an endomorphism of $E$ which is of "constant type" in the sense that the eigenvalues of $\Lambda$ are constant on each component of $\mathbf{M}$. Under these circumstances one defines a cohomology class $\underline{\varphi}(\Lambda)$ of $M$, with complex coefficients in the following manner: 


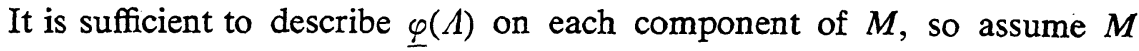
connected and let $\left\{\lambda_{i}\right\}$ denote the eigenvalues of $\Lambda$ counted with multiplicities. Also set $E(\lambda)$ equal to the largest sub-bundle of $E$ on which $(\Lambda-\lambda)$ is nilpotent. Then $E$ decomposes canonically into a direct sum of sub-bundles $E(\lambda)$ :

$$
E=\sum E(\lambda) \text {. }
$$

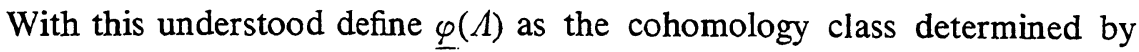
the expression

$$
\varphi\left(\lambda_{1}+x_{1}, \lambda_{2}+x_{2}, \cdots, \lambda_{q}+x_{q}\right)
$$

when $x_{i}$ is interpreted as a formal root of the Chern class of $E\left(\lambda_{i}\right)$. Because the expression (1.5) will be symmetric in all the $x_{i}$ 's which are associated to equal eigenvalues this concept makes sense and determines a class on $M$ depending on the $\left\{\lambda_{i}\right\}$ and the Chern classes of the sub-bundles $E\left(\lambda_{i}\right)$ of $E$.

For instance, when these are all line-bundles, i.e., the $\left\{\lambda_{i}\right\}$ are all distinct, one has simply:

$$
\underline{\varphi}(\Lambda)=\varphi\left(\lambda_{1}+x_{1}, \lambda_{2}+x_{2}, \cdots, \lambda_{n}+x_{n}\right) \quad x_{i}=c_{1}\left\{E\left(\lambda_{i}\right)\right\} .
$$

Note that when $M$ reduces to a point this clearly agrees with our definition of $\varphi$ as an endomorphism of a vector space. Also we recapture the expression $\underline{\varphi(E)}$ if we evaluate $\underline{\varphi}$ on the zero-endomorphism of $E$ :

$$
\underline{\varphi}\left(0_{E}\right)=\underline{\varphi}(E) \text {. }
$$

Finally, note that if det denotes the symmetric polynomial $x_{1} x_{2} \cdots x_{q}$, and $1_{E}$ is the identity endomorphism, then

$$
\underline{\operatorname{det}}\left(1_{E}\right)=c(E)=1+c_{1}(E)+\cdots+c_{m}(E) \text {, }
$$

while

$$
\underline{\operatorname{det}}\left(0_{E}\right)=c_{m}(E) \text {. }
$$

In general it is easily checked that $\operatorname{det}(\Lambda)$ will be invertible in $H^{*}(M ; C)$ if and only if $\Lambda$ is an automorphism of $E$.

With these conventions understood we may state our principal results.

Theorem 1. Let $A: \Gamma(E) \rightarrow \Gamma(E)$ be a holomorphic action of the holomorphic vector-field $X$ on the holomorphic bundle $E$ over the compact holomorphic manifold $M$. Also let $\varphi\left(x_{1}, \cdots, x_{q}\right), q=\operatorname{dim}_{C} E$, be a homogeneous symmetric polynomial of degree $e^{2}=m$, and let $N$ range over the components of the zero set of $X$. Then the local behavior of $X$ near $N$ determines certain complex numbers $\operatorname{Res}_{\varphi}(N)$ called the $\varphi$ residue of $\Lambda$ on $N$, such that

$$
\underline{\varphi}(E)[M]=\sum_{N} \operatorname{Res}_{\varphi}(N) .
$$

\footnotetext{
${ }^{2}$ Originally I asserted this formula for degree of $\varphi \leq m$, and I am greatful to P. Baum for pointing out a gap in my argument for the case deg $\varphi<m$.
} 
Here of course the left hand side denotes the evaluation of the cohomology class $\varphi(E)$ on the orientation cycle of $M$, so that for instance, the left hand side vanishes when $\operatorname{deg} \varphi<m$. When $\operatorname{deg} \varphi=\operatorname{dim}_{c} M$ and $E=T M$ is the holomorphic tangent bundle of $M$, then $\underline{\varphi}(E)[M]$ becomes a characteristic number of $M$, so that we may in general describe the left hand side of (1.8) as a characteristic number of $E$ on $M$.

This theorem was implicitly proved in [1] for the special case $E=T M$ and that proof extends directly to the present situation as will be shown in $\S 2$.

Our second theorem deals with the explicit computation of the $\varphi$-residues when $X$ vanishes nondegenerately along $N$. To describe this formula, observe first of all, that from the derivation formula (1.2) it follows immediately that on the zero set of $X, \Lambda$ becomes a differential operator of degree zero and hence induces a bundle endomorphism:

$$
\Lambda|N: E| N \longrightarrow E \mid N
$$

on each component of the zero set of $X$. By compactness this endomorphism is furthermore seen to be of constant type. Thus in particular, $\varphi(\Lambda \mid N)$ is a well determined cohomology class of $N$.

Now suppose in addition that

$N$ is a complex submanifold of $M$,

and

(1.10) the endomorphism $\theta \mid N$, induced by the action of $X$ on the holomorphic tangent bundle $T M$, along $N$ has precisely $T N$ for its kernel; i.e., the sequence

$$
0 \longrightarrow T N \longrightarrow T M|N \stackrel{\theta \mid N}{\longrightarrow} T M| N
$$

is exact.

Under these circumstances we call $N$ a nondegenerate component of zero $(X)$.

One may also state this requirement in the following manner : Clearly $\theta \mid N$ induces a homomorphism

$$
\theta^{\nu} \mid N: T M / T N \longrightarrow T M / T N
$$

on the normal bundle of $N$ in $M$. Nondegeneracy of $N$ therefore implies precisely that $\theta^{\nu} \mid N$ be an automorphism. Hence for a nondegenerate $N$, the class $\operatorname{det}\left(\theta^{\nu} \mid N\right)$ will be an invertible element of $H^{*}(M, C)$.

Now then, concerning these components we have:

Theorem 2. If $N$ is a nondegenerate component of zero $(X)$, then 


$$
\operatorname{Res}_{\varphi}(N)=\left\{\underline{\varphi}(\Lambda \mid N) / \underline{\operatorname{det}}\left(\theta^{\nu} \mid N\right)\right\} \cdot[N] .
$$

Note that when $N$ reduces to a point $P \in M$, then $\Lambda \mid P$ is simply an endomorphism of the fiber $E_{P}$ while $\theta^{\nu}|P=\theta| P$ is an endomorphism of $T_{P} M$. Thus in that case our formula specializes to:

$$
\operatorname{Res}_{\varphi}(P)=\frac{\varphi(\Lambda \mid P)}{\operatorname{det}(\theta \mid P)}=\frac{\varphi\left(\lambda_{1}, \cdots, \lambda_{q}\right)}{\nu_{1} \cdots \nu_{m}}
$$

with $\left\{\lambda_{i}\right\}$ the eigenvalues of $\Lambda \mid P$, and $\left\{\nu_{i}\right\}$ the eigenvalues of $\theta \mid P$. Finally if we set $E=T M$ and $\Lambda=\theta$, our formula (1.14) therefore goes over into the residue formula of [1].

In short our result may also be remembered this way: to pass from a point $P$ to $N$ one must construct the class

$$
\frac{\varphi\left(\lambda_{1}+x_{1}, \cdots, \lambda_{q}+x_{q}\right)}{\left(\nu_{1}+y_{1}\right)\left(\nu_{2}+y_{2}\right) \cdots\left(\nu_{m}+y_{m}\right)}
$$

in which the $x_{i}$ are formal Chern classes of the eigenbundle of $\lambda_{i}$ in $E$, and the $y_{i}$ the corresponding objects for the normal bundle to $N$, - and integrate the result over $N$. Thus eigenvalues $\lambda$ must be replaced by the sum $\lambda+x$ where $x$ is the formal Chern class associated to the eigenbundle of $\lambda$. This theorem I have found more difficult and technical than Theorem 1, and the bulk of this paper is devoted to its proof. However, the basic fact on which it hinges is closely related to the following result which might be of independent interest:

Theorem 3. Let $N$ be a nondegenerate component of zero $(X)$ and let $T M$ be equipped with a Hermitian structure (, ) which makes the decomposition

$$
T M \mid N=T N \oplus \text { Image } \theta \mid N
$$

orthogonal. Also let $S_{s}(N)$ be the normal $\varepsilon$-sphere bundle over $N$, and denote by $\sigma_{*}^{*}$ the integration over the fiber in $S_{c}(N)$. Now consider the function $|X|^{2}=(X, X)$, and set

$$
\pi=-d^{\prime} \log |X|^{2}, \quad \omega=d^{\prime \prime} \pi .
$$

Finally write

$$
\frac{\pi}{1-t \omega}=\pi\left\{1+t \omega+t^{2} \omega^{2}+\cdots\right\}
$$

Then

$$
\lim _{\varepsilon \rightarrow 0} \sigma_{*}^{\varepsilon} \frac{\pi}{1-t \omega}
$$


exists as a form on $N$ and is given by

$$
\lim _{\varepsilon \rightarrow 0} \sigma_{*}^{e}\left(\frac{\pi}{1-t \omega}\right)=(2 \pi i)^{\nu} \circ \frac{t^{\nu-1}}{\operatorname{det}(1+t k)}
$$

where $k$ is the curvature form of the normal bundle to $N$ in the induced Hermitian structure.

This result should be thought of as a geometric generalization of a wellknown formula in the cohomology of a projective bundle. Indeed let $E$ be a holomorphic vector bundle over $N$, and let $\boldsymbol{P}(E)$ be the associated projective bundle. Then if $E$ is endowed with a Hermitian structure we may consider the function $f$ on $E$ which measures the square of the height of point of $E$ from the zero section, and it is well known that the form:

$$
[\omega]=\frac{1}{2 \pi i} d^{\prime \prime} d^{\prime} \log f
$$

as interpreted on $\boldsymbol{P}(E)$, generates $H^{*}\{\boldsymbol{P}(E)\}$ over $H^{*}(N)$. In fact $1,[\omega], \cdots,[\omega]^{n-1}$ turn out to be generators of $H^{*}(\boldsymbol{P}(E))$ as an $H^{*}(N)$ module, and the Chern classes of $E$ turn out to be characterized by the equation

$$
[\omega]^{n}+[\omega]^{n-1} c_{1}(E)+\cdots+c_{n}(E)=0 .
$$

Let $\sigma_{*}$ be the integration over the fiber in this fibering. Then one finds that

$$
\sigma_{*}[\omega]^{n-1}=1
$$

from which it follows easily that qua power series in $t$,

$$
\sigma_{*}\{1 /(1-t[\omega])\}=1 / c_{t}(E) .
$$

Now, recalling that the curvature $k_{E}$ of $E$ represents the Chern class by the formula

$$
c_{t}(E)=\operatorname{det}\left(1+t i / 2 \pi \cdot k_{E}\right)
$$

the analogy of (1.15) and (1.19) is apparent. In fact, Theorem 3 can be thought of as the geometric proof of (1.19). Indeed if $X$ denotes the radial vector field on $E$ which generates the action of $R$ on $E$ given by multiplication, then the $f$ of (1.16) may easily be identified with $|X|^{2}=(X, X)$ computed in a Hermitian structure on $T E$ which is constructed out of the given structure on $E$ and the pull back of some structure on $M$. The conditions (1.9), (1.10) then become valid and so (1.15) may be used to prove (1.19).

The formula (1.15) is of course more general than (1.19). First of all $N$ need not have normal neighborhoods which are holomorphically isomorphic 
to the normal bundle of $N$, and secondly (1.15) remains meaningful in certain noncompact situations where $(1.19)$ is not.

\section{The proof of Theorem 1 reviewed}

Here we will briefly review the constructions of [3] but in the slightly extended form which is appropriate for the proof of Theorem 1.

First of all recall that $\varphi(E)$ can be represented by a differential form in the following manner. Let $\bar{E}$ be endowed with a Hermitian structure (, ). This structure then defines a canonical connection $\nabla$ on $E$ which is of type $(1,0)$ on holomorphic sections and preserves this inner product. The curvature $K=K(V)$ of this connection is then a 2 -form on $M$ with values in the bundle of endomorphisms of $E$, End $(E)$, which is characterized by the equation

$$
d^{\prime \prime} \nabla \cdot s=K(\nabla) \cdot s
$$

for a local holomorphic section of $E$. Here $d^{\prime \prime}$ denotes the $\partial / \partial \bar{z}$-part of the operator $d$ and is therefore well defined on $\Gamma(E)$.

Now our much extended $\varphi$ extends yet again naturally to a function, also denoted by $\varphi$, from forms on $W$ with values in End $E$, to ordinary forms. Thus $\varphi(K)$ is a well determined differential form on $M$ and it is a fundamental fact that the theory of characteristic classes of the form $\varphi(K)$ represents the class $(2 \pi / i)^{\operatorname{deg} \varphi} \varphi(E)$.

The basic observation in [1] was that with the aid of a holomorphic vector field $X$, one may construct a form $\eta$ on $M$-zero $X$ such that on the complement of zero $(X)$,

$$
i\left(X^{\prime}\right)\left\{\varphi(K)+d^{\prime \prime} \eta\right\}=0,
$$

where $X^{\prime}$ denotes the $(1,0)$ component of $X$ in the complexified tangent space of $M$, and $i(Y)$ the inner product with a vector field $Y$.

There, of course, the underlying bundle $E$ was taken to be $T M$, the holomorphic tangent bundle of $M$. For a more general $E$ this construction still makes sense provided $X$ admits a definite action $A$ on $E$.

Indeed under this assumption we may define an operator $L: \Gamma(E) \rightarrow \Gamma(E)$ by the formula:

$$
L \cdot s=\Lambda \cdot s-\nabla_{X} \cdot s,
$$

where $\nabla_{X}=i(X) \cdot \nabla$ is the covariant derivative of $s$ relative to our connection $\nabla$ in the direction $X$.

The operator $L$ then turns out to be of degree zero and so may be thought of as a section of the bundle End $(E): L \in \Gamma\{$ End $(E)\}$.

Note that in the zero set of $X, L$ clearly agrees with $A$, so that $L$ should be thought of as the $C^{\infty}$ extension of $\Lambda \mid$ zero $(X)$. 
Applying the operator $d^{\prime \prime}$ to (2.2) one obtains the identity

$$
d^{\prime \prime} L=i\left(X^{\prime}\right) \cdot K
$$

on which our construction hinges. Namely, just as in [3], (2.3) is first seen to imply the identity:

$$
t d^{\prime \prime} \varphi(L+t K)=i\left(X^{\prime}\right) \varphi(L+t K) .
$$

Next choose a 1 -form $\pi$ of type $(1,0)$ on $M$-zero $(X)$ such that there,

$$
\pi(X)=1 \text {. }
$$

Such a "projector" for $X$ is easy to come by and in terms of it we may construct the power-series of forms :

$$
\eta=\varphi(L+t K) \cdot \pi /(1-t \omega), \quad \omega=d^{\prime \prime} \pi .
$$

An immediate consequence of (2.4) and (2.5) is then the following Basic Identity:

$$
\varphi(L+t K)+t d^{\prime \prime} \eta-i\left(X^{\prime}\right) \eta \equiv 0 .
$$

Theorem 1 now follows from (2.7) by an application of Stoke's theorem. Indeed let $E, X, M, \Lambda$ and $\varphi$ be as in Theorem 1 and let $N$ run over the components of zero $X$. Also let $N_{\varepsilon}$ be $\varepsilon$-neighborhoods for $N$, oriented coherently with $M$, and let $\partial N_{\varepsilon}$ denote their boundaries. Assume in addition that $\operatorname{deg} \varphi=m=\operatorname{dim}_{C} M$.

We then define $\operatorname{Res}_{\varphi}(N)$ by the formula:

$$
\operatorname{Res}_{\varphi}(N)=\frac{i}{2 \pi} \lim _{t \rightarrow 0} \int_{\partial N_{e}} \varphi(L+t K) \cdot \pi /\left.(1-t \omega)\right|_{t=i / 2 \pi}
$$

so that

$$
\operatorname{Res}_{\varphi}(N)=\left(\frac{i}{2 \pi}\right)^{m} \lim _{\epsilon \rightarrow 0} \int_{\partial N_{\varepsilon}} \eta^{(m-1)}
$$

where $\eta^{(m-1)}$ is the coefficient of $t^{m-1}$ in $\eta$. Consider now the coefficient of $t^{m}$ in (2.7).

For dimensional and type reasons, one then obtains the relation

$$
\varphi(K)+d \eta^{(m-1)}=0, \quad \text { on } M \text {-zero } X \text {. }
$$

Hence by Stoke's theorem we obtain the equalities: 


$$
\begin{aligned}
\underline{\varphi}(E) & =\left(\frac{i}{2 \pi}\right)^{m} \int_{M-N_{\varepsilon}} \varphi(K)=\left(\frac{i}{2 \pi}\right)^{m} \lim _{\epsilon \rightarrow 0} \int_{M-N_{\varepsilon}} \varphi(K) \\
& =-\left(\frac{i}{2 \pi}\right)^{m} \lim _{\epsilon \rightarrow 0} \int_{M-N_{\varepsilon}} d \eta^{(m-1)} \\
& =\left(\frac{i}{2 \pi}\right)^{m} \sum \lim _{\epsilon \rightarrow 0} \int_{\delta N_{\varepsilon}} \eta^{(m-1)}=\sum_{N} \operatorname{Res}_{\varphi}(N),
\end{aligned}
$$

which establishes Theorem 1.

One may extend Theorem 1 to the case $\operatorname{deg} \varphi<m$ provided zero $(X)$ consists of nondegenerate components whose dimensions are less than the degree of $\varphi$, in the following manner:

If degree $\varphi=m-l, l>0$ define

$$
\operatorname{Res}_{\varphi}(N)=\left(\frac{i}{2 \pi}\right)^{m-\imath} \lim _{\epsilon \rightarrow 0} \int_{\partial N_{\iota}} \varphi(L+t K) \cdot \pi \omega^{l} /\left.(1-t \omega)\right|_{t=i / 2 \Pi} .
$$

Then a similar argument can be applied to the identity, obtained by multiplying (2.7) by $\omega^{l}$, to obtain the formula

$$
\left(\frac{i}{2 \pi}\right)^{m} \varphi(K) \cdot \omega^{l}(M)=\sum \operatorname{Res}_{\varphi}(N) .
$$

However, $d^{\prime \prime} \varphi(K) \cdot \pi \cdot \omega^{l-1}=\varphi(K) \cdot \omega^{l}$ so that the left hand side of (2.11) vanishes provided $\operatorname{dim} N<\operatorname{deg} \varphi$, as it should in accordance with (1.8).

\section{The proofs of Theorems 2 and 3}

It is clear from the preceding section that the proof of (1.13) hinges on an explicit evaluation of the limit $\int_{\partial N_{\iota} \eta} \eta$ as $\varepsilon \rightarrow 0$. We start therefore with some general remarks on the existence of limits of this type.

Consider then the following situation. We let $M$ be an oriented Riemannian manifold and let $N \subset M$ be an oriented connected compact submanifold of $M$. The square of the distance from $N$ is then a well defined $C^{\infty}$ function near $N$ which will be denoted by $l_{N}$ and $N_{\varepsilon}$ shall denote the $\varepsilon$-neighborhood of $N$ :

$$
N_{\iota}=\left\{p \mid l_{N}(p) \leq \varepsilon^{2}\right\} .
$$

For $\varepsilon$ small enough say $\leq \varepsilon_{0}, M^{\prime}=N_{\varepsilon_{0}}$ will then be fibered into discs by the geodesic projection

$$
\sigma: M^{\prime} \rightarrow N
$$

and $\sigma$ will furthermore induce a fibering

$$
\sigma_{\iota}: \partial N_{\epsilon} \rightarrow N,
$$


of the boundary $\partial N_{\varepsilon}$, into oriented spheres of dimension $\nu-1$, where $\nu$ is the codimension of $N$ in $M$.

We will denote the ring of $C^{\infty}$ differential forms on a manifold $M$ by $A(M)$, and indicate the induced homomorphism of a $C^{\infty}$ map by an upper asterisk. Thus

$$
\sigma_{\varepsilon}^{*}: A(N) \rightarrow A\left(\partial N_{\varepsilon}\right)
$$

is a well defined ring homomorphism.

The integration over the fiber in $\partial N_{\varepsilon} \rightarrow N$ will be denoted by $\sigma_{*}^{\varepsilon}$. Hence

$$
\sigma_{*}^{\varepsilon}: A\left(\partial N_{\varepsilon}\right) \rightarrow A(N)
$$

is an additive homomorphism only, and decreases dimensions by $(\nu-1)$. These two operations are adjoint in the sense that

$$
\int_{\partial N_{\varepsilon}} u \cdot \sigma_{\varepsilon}^{*} v=\int_{N} \sigma_{*}^{\varepsilon} u \cdot v, \quad u \in A\left(\partial N_{\varepsilon}\right), v \in A(N)
$$

and this formula characterizes $\sigma_{*}^{c}$.

Suppose now that $\eta$ is a form defined on $M^{\prime}-N$, and let $\iota_{\varepsilon}: \partial N_{t} \rightarrow M$ be the injection. For $\varepsilon<\varepsilon_{0}$ we may then construct the form $\sigma_{*}^{c} \cdot \ell_{\varepsilon}^{*} \eta$ on $N$, and consider the limit

$$
\sigma_{*}(\eta)=\lim _{\varepsilon \rightarrow 0} \sigma_{*}^{\varepsilon} \cdot \iota_{c}^{*} \eta
$$

Clearly if this limit exists as a $C^{\infty}$ form on $N$, then

$$
\lim _{\varepsilon \rightarrow 0} \int_{\partial N_{\varepsilon}} \eta=\int_{N} \sigma_{*}(\eta)
$$

Under these circumstances we therefore say that

$$
\lim _{\varepsilon \rightarrow 0} \int_{\partial N_{\varepsilon}} \eta
$$

exists locally on $N$, and refer to $\sigma_{*}(\eta)$ as the principal value of $\eta$ on $N$.

We will need a criterion for the existance of principal value for $\eta$ when $\eta$ is of the type

$$
\eta=\mu / f^{n}
$$

with $\mu \in A\left(M^{\prime}\right)$ and $f$ a $C^{\infty}$-function on $M^{\prime}$ which is non-negative and assumes its nondegenerate minimum on $N$. Thus $f$ has the properties:

$$
f \geq 0 \text { on } M^{\prime}, \quad f^{-1}(0)=N
$$

and $f$ has no critical values other than 0 on $M^{\prime}$. 
On $N$ the Hessian of $f$ has rank $=\operatorname{codim} N$.

Following Morse we will call functions of this type "neighborhood functions" of $M$. Note that the function $l_{N}$ of course satisfies these conditions and is in fact the prototype of a neighborhood function for $M$.

To state our presumably well known criterion, consider the normal Taylor expansion for $\mu \in A\left(M^{\prime}\right)$ at a point $P \in N$. Such an expansion is constructed as follows.

Let $\left\{x_{\nu+1}, \cdots, x_{m}\right\}$ be a coordinate system on $N$ centered at $P$ and valid in a patch $U>P$ on $N$. Also let $y_{1}, \cdots, y_{\nu}$ be $C^{\infty}$ functions on $U^{\prime}=\sigma^{-1}(U)$, so that

$$
\left(y_{1}, \cdots, y_{\nu}, \sigma^{*} x_{\nu+1}, \cdots, \sigma^{*} x_{m}\right)
$$

form a coordinate system over $U^{\prime}$ with respect to which $N$ is described the conditions $y_{1}=\cdots=y_{\nu}=0$.

We may now take the Taylor expansion of $\mu$ relative to the coordinates (3.5), and lump the $x$ terms together to obtain an expansion of the form:

$$
\mu=\sum y^{\alpha} d y^{\beta} \cdot \sigma^{*} \mu_{\alpha \beta}
$$

with $\mu_{\alpha \beta} \in A(N)$ while $y^{\alpha}$ and $d y^{\beta}$ are short for $y_{1}^{\alpha_{1}} \ldots y_{\nu}^{\alpha_{\nu}}$ and $d y_{1}^{\beta_{1}} \ldots d y_{\nu}^{\beta_{\nu}}$, the $\alpha_{i}$ being non-negative integers, while the $\beta_{i}$ range over the integers 0 and 1 .

Under the substitution $y_{i} \rightarrow \lambda y_{i}$ the various terms of (3.6) pick up different powers of $\lambda$, and so break up this series into components $\mu^{(k)}$ of normal homogeneity $k$;

$$
\mu=\sum_{k \geq 0} \mu^{(k)}
$$

Thus if we set $|\alpha|=\sum \alpha_{i}$, then

$$
\mu^{(k)}=\sum_{|\alpha+; \beta|=k} y^{\alpha} d y^{\beta} \sigma^{*} \mu_{\alpha \beta} .
$$

We now say that $\mu$ vanishes to order $n \bmod N$ at $P$ if all the $\mu^{(k)}$ with $k<n$ vanish at $p$. This condition is seen to be independent of the normal coordinates chosen-indeed, also independent of the projection $\sigma$, and if $\mu$ vanishes to order $n$ at all $P \in N$ we say simply that $\mu$ vanishes to order $n \bmod N$. We also record this fact by the notation:

$$
\mu \equiv 0 \quad \bmod N^{n} .
$$

Note that "vanishing $\bmod N^{n}$ " is a purely $C^{\infty}$ notion, and as remarked above, independent of the projection $\sigma: M^{\prime} \rightarrow N$.

With these conventions understood one has the following proposition:

Proposition 3.1. Let $\sigma: M^{\prime} \rightarrow N$, be as above and let $\mu$ be $a C^{\infty}$ form in $A\left(M^{\prime}\right)$. If $\mu$ vanishes to order $2 n$ relative to $N$ : 


$$
\mu \equiv 0 \quad \bmod N^{2 n}
$$

then the limit

$$
\sigma_{*}\left(\mu / f^{n}\right)=\lim _{\varepsilon \rightarrow 0} \sigma_{*}^{\varepsilon} \cdot \iota_{\iota}^{*}\left(\mu / f^{n}\right)
$$

exists for every neighborhood function $f$ of $N$. In fact if near $P, \mu$ is given by

$$
\mu=\sum y^{\alpha} d y^{\beta} \cdot \sigma^{*} \mu_{\alpha \beta}
$$

then

$$
\sigma_{*}\left(\mu / f^{n}\right)_{P}=\Sigma^{\prime}\left[\int_{l_{P}^{(2)}=1} \frac{y^{\alpha} d y^{\beta}}{\left[f_{P}^{(2)}\right]^{k}}\right] \mu_{\alpha \beta_{P}}
$$

where $\Sigma^{\prime}$ denotes the sum over those $\alpha, \beta$ with

$$
\begin{aligned}
|\alpha|+|\beta| & =2 n, \\
|\beta| & =\nu-1,
\end{aligned}
$$

and the integral is taken over the ellipsoid $l_{P}^{(2)}(y)=1$ in $\boldsymbol{R}^{\nu}$.

The proof of this proposition is quite straightforward. Near $P$ let

$$
\mu=\mu^{(2 n)}+\mu^{(2 n+1)}+\cdots
$$

be a normal series for $\mu$ and let

$$
f=f^{(2)}+f^{(3)}+\cdots, l_{N}=l^{(2)}+l^{(3)}+\cdots
$$

be the normal series of $f$ and $l$. That these start with the second order terms is of course a direct consequence of the conditions (3.3) and (3.4). They imply furtheremore, that the quadratic forms in $y: f_{P}^{(2)}$ and $l_{P}^{(2)}$ are positive definite. Hence by an easy extension of a lemma of Morse, we may find normal coordinates $\left\{y_{i}\right\}$ for $N$ near $P$ so that there

$$
l_{N}=\sum y_{j}^{2} .
$$

In these coordinates, $\partial N_{\varepsilon}$ is given by the equation

$$
\sum y_{j}^{2}=\varepsilon^{2} \text {. }
$$

Hence $f_{(P)}^{(2)}>0$ on $\partial N_{\varepsilon}$ so that near $P, \mu / f^{n}$ is uniformly and arbitrarily well approximated by the partial sums of a series of the type:

$$
\frac{\mu^{(2 n)}}{\left[f^{(2)}\right]^{n}}+\frac{c_{1}}{\left[f^{(2)}\right]^{n+1}}+\frac{c_{2}}{\left[f^{(2)}\right]^{n+2}}+\cdots
$$


the normal degree of $c_{j}$ exceeding $2(n+j)$. Thus the terms involving $c_{j}$ are homogeneous of order $>0$.

Now the operation $\sigma_{*}^{\varepsilon}$ consists of integrating "relative to the $y$ coordinates" over the sphere (3.9). Hence all these terms disappear as $\varepsilon \rightarrow 0$, while the leading term which is homogeneous of degree zero stays constant and has a finite value given by integrating over the unit sphere say, in the $y_{j}$ 's. The formula (3.7) follows directly.

With this material out of the way let us return to our problem of computing the $\varphi$-weight of a nondegenerate manifold $N$ in the zero set of a holomorphic vector field $X$. We will therefore assume the conditions and the terminology of the preceding sections.

Note first that our form $\eta=\varphi(L+t K) \cdot \pi /(1-t \omega)$ is the product of the smooth form $\varphi(L+t K)$, defined on all of $M$, and the form $\pi /(1-t \omega)$ which becomes singular on $N$.

Our plan is therefore to construct a projector $\pi$ for $X$ such that $\pi /(1-t \omega)$ will satisfy the conditions of our lemma and hence have a principal value on $N$. Thereafter it follows directly from the permanence law (3.1) that

$$
\sigma_{*}\{\eta\}=\iota^{*} \varphi(L+t K) \cdot \sigma_{*}(\pi /(1-t \omega))
$$

where $\iota^{*}$ denotes the restriction to $N$. Hence under this assumption one obtains the expression

$$
\operatorname{Res}_{\varphi}(N)=\left.\frac{i}{2 \pi} \int_{N} \iota^{*} \varphi(L+t K) \cdot \sigma_{*}\{\pi /(1-t \omega)\}\right|_{t=i / 2 \pi} .
$$

It should be stressed here that $\sigma_{*}(\pi /(1-t \omega))$ need not exist for a general projector $\pi$, although the limit of $\int_{\partial N \iota} \eta^{(m-1)}$ will of course still exist.

To construct such a good "projector" $\pi$ for $X$ one proceeds in the following manner. Recall that the action $\theta(X)$ of $X$ on $T M$ induces a splitting

$$
T M \mid N=T N \oplus \text { image } \theta \mid N
$$

along $N$. First we choose a Hermitian structure on $T M$ which makes this decomposition orthogonal, and denote by $\delta$ the connection which it induces on $T M$. Next we construct the analogue of $L$, that is, an endomorphism

$$
A: T M \rightarrow T M
$$

defined by

$$
A \cdot s=\theta(X) \cdot s-\delta_{X} \cdot s
$$

One then has the following analogue of Theorem 3.

Theorem 4. Let $\pi$ denote the form of type $(1,0)$ given by 


$$
\pi=-\left(\delta^{\prime} X^{\prime}, A X^{\prime}\right) /\left|A X^{\prime}\right|^{2}
$$

where $A$ and $\delta$ are as above, and the prime denotes the type $(1,0)$ part of $\delta$ and $X$. Then $\pi$ is a projector for $X$ in a suitable neighborhood $\bar{M}$ of $N$, and the form $\pi /(1-t \omega), \omega=d^{\prime \prime} \pi$ has a principal value on $N$ which is given by

$$
\sigma_{*}(\pi /(1-t \omega))=(-2 \pi i)^{\nu} t^{\nu-1} / \operatorname{det}\left(\theta^{\nu}+t k^{\nu}\right) .
$$

Here $\theta^{\nu}$ is the endomorphism induced by $\theta(X)$ on the normal bundle of $N$ in $M$, and $k^{\nu}$ denotes the curvature of the normal bundle to $N$ relative to the connection induced on it by $\delta$.

Before we give a proof of this theorem we will show that Theorem 2 follows from it directly. For this purpose we first extend the $\pi$ given by (3.13) to a projector for $X$ on all of $M$-zero $X$. Thereafter combining (3.10) with (3.14) we obtain the expression

$$
\operatorname{Res}_{\varphi}(N)=\int_{N} \iota^{*} \varphi(L+t K) /\left.\operatorname{det}\left(\theta^{\nu}+t k^{\nu}\right)\right|_{t=i / 2 \pi}
$$

whose right hand side clearly represents

$$
\left\{\underline{\varphi}(E \mid N) / \underline{\operatorname{det}}\left(\theta^{*} \mid N\right)\right\}(N)
$$

and so establishes the

Corollary. Theorem 2 is valid.

We come now to the proof of Theorem 4. First it therefore has to be checked that $\pi$ is a projector for $X$ in some vicinity of $N$. Now $A$ extends the endomorphism $\theta \mid N$ and hence, because of our nondegeneracy assumption, is nonsingular in the normal direction to $N$. Thus in a suitable $N_{\varepsilon},|A X|^{2}$ will vanish only on $N$ and in fact will be a neighborhood function for $N$. Thus $\pi$ is well defined near but away from $N$. Clearly in this region

$$
\pi(X)=-\left(\delta_{X}\left(X^{\prime}\right), A X^{\prime}\right) /\left|A X^{\prime}\right|^{2} .
$$

Hence (3.12) and the fact that $\theta(X) \cdot X^{\prime}=0$ imply that $\pi(X)=1$. Thus $\pi$ is a projector for $X$.

Next we turn to the principal value of $\pi /(1-t \omega)$. We must therefore estimate the forms $\pi \cdot \omega^{q-1}, q=1,2, \ldots$. To derive an expression for this form from (3.13) we use the invariance of the inner product under $\delta$. Also to simplify life we will drop the prime on $X$ and until further notice write simply $X$ for $X^{\prime}$-the type $(1,0)$ component of $X$. Now then, it follows that

$$
\begin{aligned}
\pi \cdot \omega^{q-1} & =-\left(\delta^{\prime} X, A X\right)\left\{-\left(\delta^{\prime \prime} \delta^{\prime} X, A X\right)+\left(\delta^{\prime} X, \delta^{\prime} A X\right)\right\}^{q-1} \cdot|A X|^{-2 q} \\
& =(-1)^{q}\left(\delta^{\prime} X, A X\right) \cdot\left\{(k X, A X)+\left(\delta^{\prime} X, \delta^{\prime} A X\right)\right\}^{q-1} \cdot|A X|^{-2 q}
\end{aligned}
$$

where $k$ denotes the curvature of $\delta$. 
We claim that the numerator (3.16) is congruent to zero $\bmod N^{2 q}$. Indeed we clearly have

$$
X \equiv 0 \quad \bmod N^{1},
$$

if we extend our $\bmod N$ notation to forms with values in $T M$ in the natural way, so that such a form vanishes $\bmod N^{n}$ if and only if all its components relative to a local $C^{\infty}$ frame for $T M$ vanish $\bmod N^{n}$.

The derivation property of $\delta$ then implies that

$$
\delta^{\prime} X \equiv 0 \quad \bmod N^{1},
$$

and in fact also that the other terms $k X, \delta^{\prime} A X$ appearing in inner products of the numerator vanish mod $N^{1}$. The individual inner products are therefore $0 \bmod N^{2}$ and our assertion is immediate.

Applying the criterion of Proposition 3.1 it follows that $\pi \omega^{q-1}$ has a principal value along $N$ so that $\sigma_{*}(\pi /(1-t \omega))$ is well defined and could be computed according to (3.7). Up to this point the orthogonality of the decomposition (3.11) was not used. Hence $\pi /(1-t \omega)$ always has a principal value on $N$, although the value would be quite difficult to determine in general.

However under our assumptions one also has the following

Lemma. Let $N$ have codimension $\nu$ in $M$ and set

$$
\Omega=(-1)^{\nu}\left(\delta^{\prime} X, A X\right) \cdot\left(\delta^{\prime} X, \delta^{\prime} A X^{\nu-1}\right) .
$$

Then under our hypothesis on $\pi$,

$$
\sigma_{*}\left(\pi \omega^{q-1}\right)=(-1)^{q-\nu}\left(\begin{array}{c}
q-1 \\
q-\nu
\end{array}\right) \sigma_{*}\left\{\frac{\Omega(k X, A X)^{q-\nu}}{(A X, A X)^{q}}\right\} .
$$

In particular, $\sigma_{*}\left(\pi \omega^{q-1}\right)=0$ for $q<\nu$, and qua power series in $t$,

$$
\sigma_{*}(\pi / 1-t \omega)=t^{\nu-1} \sigma_{*}\left[\Omega /\left\{|A X|^{2}+t(k X, A X)\right\}^{*}\right] .
$$

To establish (3.19) all the other terms in the binomial expansion of (3.16) have to be eliminated. For this purpose consider the nature of $X$ near $P \in N$ in greater detail.

Because $N$ is a holomorphic variety we can find a coordinate patch $U$ centered at $P$, with holomorphic coordinates $\left\{z_{1}, \cdots, z_{m}\right\}, m=\operatorname{dim}_{c} M$ such that

$$
N \cap U=\left\{q \in U \mid z_{1}(q)=\cdots=z_{\nu}(q)=0\right\}
$$

$\nu$ being the codimension of $N$ in $M$.

Because $X$ is nondegenerate near $N$ one can furthermore choose these coordinates in such a manner that on $U$ 


$$
X \equiv-\sum z_{\alpha} X_{\beta \alpha} \frac{\partial}{\partial z_{\beta}} \bmod N^{2},
$$

with $\alpha, \beta$ ranging over the integers 1 to $\nu$, while $\left\|X_{\alpha \beta}\right\|$ is a nonsingular matrix of holomorphic functions.

Note that from (3.21) it follows directly that

$$
\begin{array}{lll}
\theta(X): \frac{\partial}{\partial z_{\alpha}} \equiv \sum_{\beta=1}^{\nu} X_{\alpha \beta} \frac{\partial}{\partial z_{\beta}} & \bmod N^{1} & 1 \leq \alpha \leq \nu \\
\theta(X): \frac{\partial}{\partial z_{j}} \equiv 0 & \bmod N^{1} & \text { if } \quad j>\nu .
\end{array}
$$

Hence the matrix $\left\|X_{\alpha \beta}\right\|$ represents $A$ along $N$ :

$$
\left.A \frac{\partial}{\partial z_{\alpha}}\right|_{N}=\left.\sum_{\beta} X_{\beta \alpha} \frac{\partial}{\partial z_{\beta}}\right|_{N}
$$

whence, finally $\frac{\partial}{\partial z_{\alpha}}(\alpha=1, \cdots, \nu)$ are in the image of $\theta \mid N$ while the remaining $\frac{\partial}{\partial z_{j}}$ restrict to elements in the kernel of $\theta \mid N$. Hence

$$
\left(\frac{\partial}{\partial z_{\alpha}}, \frac{\partial}{\partial z_{j}}\right) \equiv 0 \bmod N^{1}, \quad 1 \leq \alpha \leq \nu<j,
$$

from which it follows that the connection forms $\Theta_{i j}$ of $\delta$ relative to our base $\left\{\frac{\partial}{\partial z_{i}}\right\}$ satisfy the condition

$$
\Theta_{\alpha j} \equiv 0 \bmod N^{1}
$$

in the same range of indices: $1 \leq \alpha \leq \nu<j \leq m$. Indeed the special nature of $\delta$ implies that the whole connection matrix $\Theta$ is expressed in terms of the matrix $H$ of inner product $\left(\frac{\partial}{\partial z_{i}}, \frac{\partial}{\partial z_{j}}\right)$ by the formula $\Theta=d^{\prime} H \cdot H^{-1}$, so that (3.25) follows from (3.24) and the fact that $d^{\prime}$ preserves the order of vanisning $\bmod N$.

Now, then, consider the covariant derivative $\delta X$. One has

$$
\delta X \equiv-\Sigma\left\{d\left(z_{\alpha} X_{\alpha \beta}\right) \otimes \frac{\partial}{\partial z_{\beta}}+z_{\alpha} X_{\alpha \beta} \Theta_{\beta j} \otimes \frac{\partial}{\partial z_{j}}\right\} \bmod N^{2},
$$

with $\alpha, \beta$ ranging over $1, \cdots, \nu$, while $j$ ranges from 1 to $m$. Applying (3.25) we see that the components of $\delta X$ in the tangential direction to $N$ vanish $\bmod N^{2}$ so that one finally obtains the expression 


$$
\delta X \equiv \sum_{\alpha=1}^{2} \rho_{\alpha} \frac{\partial}{\partial z_{\alpha}} \bmod N^{2}
$$

where the $\rho_{\alpha}$ are certain 1 -forms, $\nu$ in number. It follows that $\bmod N^{3}$ both $\left(\delta^{\prime} X, \delta^{\prime} A X\right)$ and $\left(\delta^{\prime} X, A X\right)$ are in the ideal generated by these $\nu 1$-forms so that

$$
\left(\delta^{\prime} X, \delta^{\prime} A X\right)\left(\delta X, \delta^{\prime} A X\right)^{\nu-1+s} \equiv 0 \bmod N^{2(\nu+s)+1}
$$

whenever $s>0$. But then no terms which involve $\left(\delta^{\prime} X, \delta^{\prime} A X\right)$ to a power greater than $(\nu-1)$ can contribute to $\sigma_{*}\left(\pi \omega^{q-1}\right)$.

On the other hand terms involving this form to a smaller power do not contain sufficiently many "normal differentials" to enter nontrivially into the integration. Indeed let

$$
k=k^{(0)}+k^{(1)}+\cdots
$$

be the normal expansion of $k$ relative to real coordinates $\left\{y_{i}\right\}, i=1, \cdots, 2 \nu$, given by the real and imaginary parts of $z_{1}, \cdots, z_{\nu}$. Then $k^{(0)}$ involves no $d y$ 's. Further we clearly have

$$
(k X, A X) \equiv\left(k^{0} X, A X\right) \bmod N^{3} .
$$

Hence we may replace $(k X, A X)$ by $\left(k^{0} X, A X\right)$ in (3.19) and then our assertion is self evident.

This completes the proof of (3.19). To pass from there to (3.20) is then a purely formal matter.

At this stage then, the proof of Theorem 4 is reduced to the derivation of the formula:

$$
\sigma_{*}\left[\Omega \cdot\{(A X, A X)+t(k X, A X)\}^{-\nu}\right]=(-2 \pi i)^{\nu} / \operatorname{det}\left(\theta^{\nu}+t k^{\nu}\right),
$$

and this relation now follows directly from our algorithm (3.7) once we make the following observations.

Consider $C^{\nu}$ with coodinates $z_{1}, \cdots, z_{\nu}$. Also write $($,$) for the usual$ Hermitian inner product in $C^{\nu}$, and write $v$ for the differential form

$$
v(z)=(-1)^{\nu}(d z, z)\{(d z, d z)\}^{\nu-1} .
$$

Then the following holds:

Lemma. Let $a: C^{\nu} \rightarrow C^{\nu}$ be an endomorphism and define a formal powerseries $\psi(1+t a)$ in $t$ by the formula

$$
\psi(1+t a)=\int_{\{z \mid=1} \frac{\nu(z)}{\{(z, z)+t(a z, z)\}^{\nu}}
$$


where the integrand is to be first expanded in $t$ and then integrated over the unit sphere in $C^{\nu}$. Then $\phi$ is given by the formula

$$
\psi(1+t a)=\psi(1) \cdot \frac{1}{\operatorname{det}(1+t a)} .
$$

\section{Furthermore}

$$
\phi(1)=(-2 \pi i)^{\nu} .
$$

Proof. Let $\mathrm{H}^{+}$denote the positive definite self adjoint transformations of $C^{\nu}$. For $p \in H^{+}$the integral

$$
\phi(p)=\int_{|z|=1} \frac{v(z)}{\{(p z, z)\}^{\nu}}
$$

is well defined. Further the rational form $v(z) \cdot(p z, z)^{\nu}$, which is clearly holomorphic in $C_{\nu}-0$ is seen to be closed there:

$$
d\left\{v(z) \cdot(p z, z)^{-\nu}\right\}=0 .
$$

If we change variables in (3.32) by setting $z=n z^{\prime}$, where $n$ is an automorphism of $C^{\nu}$, then $\psi$ is given by

$$
\phi(p)=\int_{\left|n z^{\prime}\right|=1} \frac{v\left(n z^{\prime}\right)}{\left(p n z^{\prime}, n z^{\prime}\right)} .
$$

Now $v\left(n z^{\prime}\right)=\operatorname{det}\left(n \cdot n^{*}\right) \cdot v$ where $n^{*}$ is the adjoint to $n$, as is easily checked. Hence

$$
\phi(p)=\operatorname{det}\left(n \cdot n^{*}\right) \int_{\left(n z^{\prime}\right)=1} \frac{v\left(z^{\prime}\right)}{\left(n^{*} p n z^{\prime}, z^{\prime}\right)}
$$

However the surface $\left|n z^{\prime}\right|=1$ is homotopic to the surface $|z|=1$ in $C^{n}-0$, whence by (3.33), we may replace this surface $\left|n z^{\prime}\right|=1$ by the unit sphere in the integral (3.34). There results the identity

$$
\phi(p)=\operatorname{det}\left(n \cdot n^{*}\right) \phi\left(n p n^{*}\right)
$$

valid for all $p \in H^{+}$. To proceed further recall that every $p \in H^{+}$may be written in the form

$$
p=m m^{*}
$$

with $m$ nonsingular and even in $H^{+}$. Setting $n=m^{-1}$ in (3.35) one therefore obtains the identity 


$$
\phi(p)=\frac{1}{\operatorname{det} p} \cdot \phi(1)
$$

valid on $H^{+}$.

Next let $p \in H^{+}$and let $h$ be an arbitrary self adjoint transformation. Then for small real $t$

$$
(p+t h) \in H^{+} .
$$

Hence (3.35) implies that qua power series in $t$,

$$
\psi(p+t h)=\frac{1}{\operatorname{det}(p+t h)} \cdot \phi(1) .
$$

On the other hand the power series $\psi(p+t h)$ depends on $h$ in an algebraic manner. Hence the identity (3.35) is valid also when $h$ is replaced by $i h$; $i=\sqrt{-1}$.

Now finally let $a$ be an arbitrary transformation. We may then decompose it in the form

$$
a=a^{+}+i a^{-}
$$

where $a^{+}$and $a^{-}$are Hermitian. Hence for small real $t$ we have the numerical identity

$$
\psi\left\{\left[p+t a^{+}\right]+t i a^{-}\right\}=\frac{1}{\operatorname{det}(p+t a)} \cdot \psi(1)
$$

from which the desired formal identity (3.30) follows.

We leave (3.31) to the reader; the formula follows from

$$
v(z)=(-1)^{\nu}(\nu-1) ! \sum_{\alpha} d z_{1} d \bar{z}_{1} \cdots d z_{\alpha} \bar{z}_{\alpha} \cdots d z_{\nu} d \bar{z}_{\nu}
$$

and the usual formula for the volume of $S^{2 \nu-1}$.

Let us return now to the proof of (3.28) and so complete the proof of Theorem 2.

Using the coordinates $z_{\alpha}$ of (3.21) and the criterion (3.1) it is then easy to see that the value of $\sigma_{*}\left[\Omega\left\{|A X|^{2}+t(k X, A X)\right\}^{-2}\right]$ at $P$ is given by the integral,

$$
\int \frac{(-1)^{\nu}\left(A d z, A^{2} z\right)\left(A d z, A^{2} d z\right)^{\nu-1}}{\left\{\left(A^{2} z, A^{2} z\right)+t\left(k A z, A^{2} z\right)\right\}^{\nu}}
$$

over the unit sphere in the $z$ 's where $A$ and $k$ stand for the normal components of $A$ and $k$ at $P$. Now replacing $z$ by $A^{2} z$ this integral transforms to 


$$
\int \frac{(-1)^{\nu}\left(A^{-1} d z, z\right)\left(A^{-1} d z, d z\right)^{\nu-1}}{\left\{(z, z)+t\left(k A^{-1}, z, z\right)\right\}}
$$

and hence is equal to

$$
\frac{1}{\operatorname{det} A^{-1}} \cdot \psi\left(1+t k A^{-1}\right)=\frac{1}{\operatorname{det}(A+t k)} \psi(1) .
$$

Recalling that $A$ represents $\theta \mid N$ along $N$ we therefore obtain the desired formula (3.28).

Theorem 3 can clearly be proved in a quite similar manner.

\section{Bibliography}

[1] M. Atiyah \& I. Singer, The index of elliptic operators. III, to be published.

[2] M. Atiyah \& R. Bott, A Lefschetz fixed point formula for elliptic differential operators, Bull. Amer. Math. Soc. 72 (1966) 245-250.

[3] R. Bott, Vector fields and characteristic numbers, Michigan Math. J. 14 (1967) 231244.

[4] L. Illusie, Manuscript in preparation.

HARVARD UNIVERSITY 